\title{
Real-time currency recognition on video using AKAZE algorithm
}

\author{
Faisal Dharma Adhinata*), Rifki Adhitama, Alon Jala Tirta Segara \\ Department of Software Engineering, Faculty of Informatics, Institut Teknologi Telkom Purwokerto \\ Jl. D. I. Panjaitan No. 128, Purwokerto, Jawa Tengah 53147, Indonesia
}

\begin{abstract}
How to Cite: F. D. Adhinata, R. Adhitama, and A. J. T. Segara, "Real-time currency recognition on video using AKAZE algorithm," Jurnal Teknologi dan Sistem Komputer, vol. 9, no. 4, pp. 191-198, 2021. doi: 10.14710/jtsiskom.2021.13970, [Online].
\end{abstract}

\begin{abstract}
Currency recognition is one of the essential things since everyone in any country must know money. Therefore, computer vision has been developed to recognize currency. One of the currency recognition uses the SIFT algorithm. The recognition results are very accurate, but the processing takes a considerable amount of time, making it impossible to run for realtime data such as video. AKAZE algorithm has been developed for real-time data processing because of its fast computation time to process video data frames. This study proposes the faster real-time currency recognition system on video using the $A K A Z E$ algorithm. The purpose of this study is to compare the SIFT and $A K A Z E$ algorithms related to a real-time video data processing to determine the value of F1 and its speed. Based on the experimental results, the $A K A Z E$ algorithm is resulting $F 1$ value of 0.97 , and the processing speed on each video frame is 0.251 seconds. Then at the same video resolution, the SIFT algorithm results in an $F 1$ value of 0.65 and a speed of 0.305 seconds to process one frame. These results show that the AKAZE algorithm is faster and more accurate in processing video data.
\end{abstract}

Keywords - currency recognition; SIFT algorithm, AKAZE algorithm; real-time video data

\section{INTRODUCTION}

Object recognition is the process of identifying objects based on the characteristics of an object in a digital image or video. The characteristics of an object are often called features of the object. There is a feature extraction stage in image or video data processing. The human eye can easily recognize an object, but the computer requires several features to process, such as the color, size, and shape of an object [1]. Object recognition using computers has developed in everyday life, including the recognition of aircraft and ships [2], recognition of butterflies, ants, cameras, and faces [3], and also currency recognition [4]. One of the objects that the researcher developed is currency objects. The recognition of currency objects is beneficial because everyone knows money. Even those who are illiterate can recognize the type of money.

\footnotetext{
*) Corresponding author (Faisal Dharma Adhinata) Email: faisal@ittelkom-pwt.ac.id
}

Some of the techniques developed in currency recognition are template matching [4] and machine learning [1]. In the template matching technique, the stage that most influences the object recognition result is feature extraction. The feature extraction algorithm greatly determines the accuracy and speed of object matching, especially in the video data processing. Video data processing is done by extracting the video into frames. Object recognition is done by extracting the features contained in the object. Two types of features are extracted from the frame or image, namely local feature [5] and global feature [6]. Global features are usually used to detect objects and classify them. Instead, local features are used for object recognition or identification.

Some of the local feature extraction algorithms are SIFT [7], SURF [8], ORB [9], and AKAZE [10]. Research by Jing Xu et al. [4] introduced currency coin recognition using the SIFT algorithm. The research results are very accurate, but the matching takes 0.59 seconds. This speed makes the system unable to run in real-time processing. Furthermore, researchers have also solved the currency recognition problem by using deep learning techniques [11], [12]. The use of deep learning requires the data to be trained in advance for a long time. Therefore, another technique that does not involve training in currency recognition is needed.

Several studies used the AKAZE template matching algorithm in different case studies. Research by Kuznetsov and Savchenko [5] used the AKAZE algorithm to detect logos of sports teams. Using the AKAZE algorithm for a matching logo results in a more optimal $F 1$ value than other feature extraction algorithms. AKAZE algorithm only spends 0.15 seconds to process each video frame [13]. Using AKAZE in previous research resulted in the optimal value of $F 1$ and speed.

This study proposes the use of the AKAZE algorithm for real-time currency recognition. This research will use a template matching approach with a suitable method for real-time processes with no training process as in the stages of deep learning. For comparison, we also use the SIFT algorithm to compare the $F 1$ value and speed to the AKAZE algorithm. In the end, we will discuss the suitable algorithm for the case of real-time currency recognition on video.

This paper is organized into four sections. The first section is an introduction, while section 2 describes the 
methods used in this study. Next, section 3 discusses the results and evaluation of this system. The last section contains conclusions and suggestions for further work.

\section{RESEARCH METHODS}

The currency recognition system starts with acquiring video data and the query image. Video data is extracted into frames for the next stage of processing. The large query image is resized to be matched with the currency object contained in the video. Then, both query image and video frames are carried out by feature extraction using the SIFT or AKAZE algorithm. The results of feature extraction are keypoints and descriptors of features.

Keypoints are unique coordinate points as object features, while descriptors are numbers that define keypoints. The next stage is matching the descriptor in the query image and the video frame. The matching features of the SIFT algorithm use the FLANN method, while the AKAZE algorithm uses the Brute-Force Matcher (BFMatcher) method. The result of feature matching is done by forming a polygon using a homography matrix. If the polygons are formed and the query images with video frames match, there is a currency object corresponding to the input query image. The architecture of the currency recognition system is shown in Figure 1.

\section{A. Data acquisition}

Data acquisition is divided into two parts: query image acquisition and video data acquisition. The query image uses Indonesian paper currency with a nominal value of 1000, 2000, 5000, 20000, and 50000 Rupiahs, as shown in Figure 2. The video data acquisition, in this case, uses a 2 MP HiLook camera with Full HD resolution and ten fps. The number of video frames used for the experiment is 600 video frames. For each nominal amount, we use three videos with a distance of $10 \mathrm{~cm}$ and $30 \mathrm{~cm}$. Meanwhile, the video recording applied two resolutions, Full HD and HD. Table 1 shows the number of videos used in this research. The total number of videos is 20 videos.

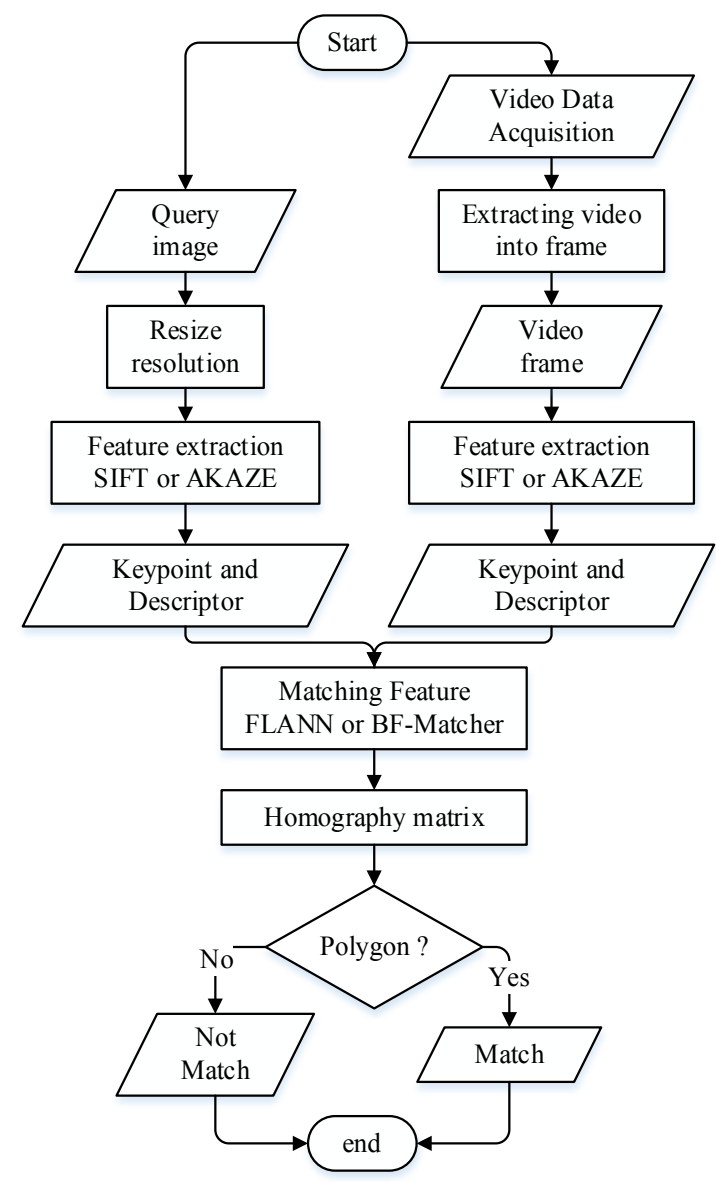

Figure 1. The architecture of the currency recognition system

Table 1. Details of the data in this research

\begin{tabular}{cccc}
\hline No & $\begin{array}{c}\text { Recorded } \\
\text { distance (cm) }\end{array}$ & Resolution & Video currency \\
\hline 1 & 10 & Full HD & $1000,2000,5000$, \\
& & & 20000, and 50000 \\
2 & 10 & HD & $1000,2000,5000$, \\
& & & 20000, and 50000 \\
3 & 30 & Full HD & $1000,2000,5000$, \\
& \multirow{2}{*}{30} & HD & 20000, and 50000 \\
4 & 30 & & $2000,2000,5000$, and 50000 \\
\hline
\end{tabular}
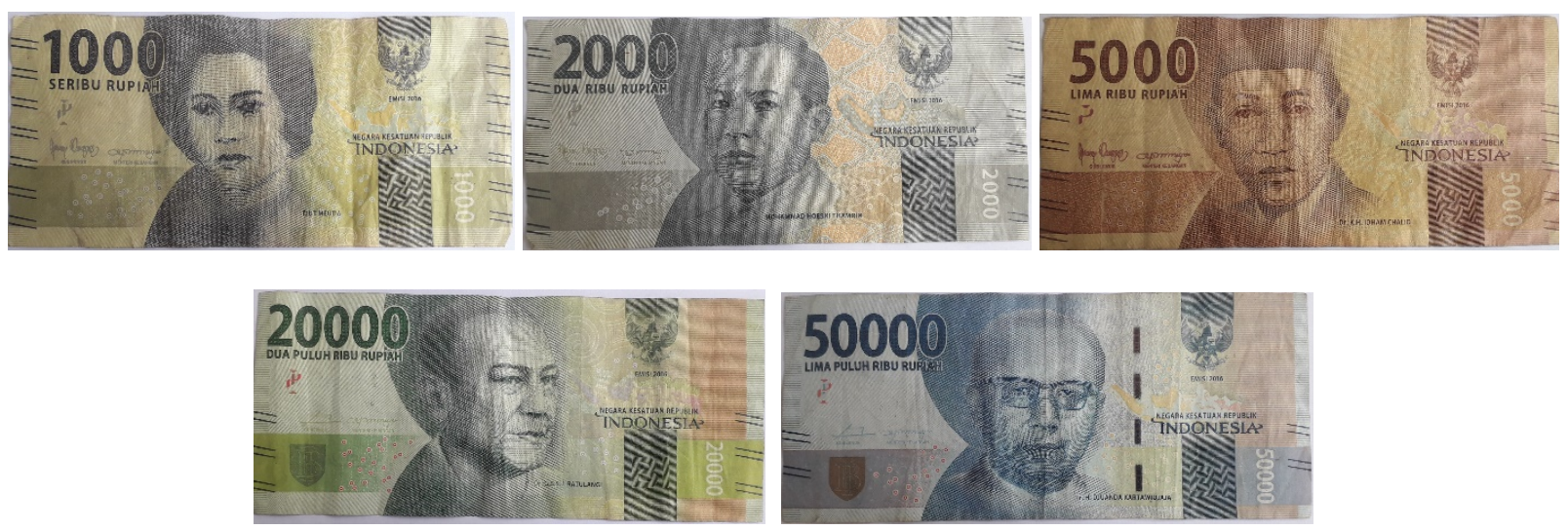

Figure 2. Indonesian paper currency for query image input 


\section{B. SIFT algorithm}

The SIFT algorithm consists of four stages: searching extreme values on scale-space, detecting keypoints, determining orientation, and creating keypoint descriptors [7]. The flowchart of SIFT algorithm is shown in Figure 3. The first stage is constructing a scale-space (octave) using Gaussian blur using (1). $L$ is a blurred image. Then, $G$ is the Gaussian Blur operator. $I$ is an image where $x, y$ is the location coordinates. $\sigma$ is the scale parameter as the amount of blur. The $*$ is the convolution operation in $x$ and $y$. It applies Gaussian blur $G$ onto the image $I$. The SIFT algorithm on each detection requires four octaves and five blur scales.

$$
L(x, y, \sigma)=G(x, y, \sigma) * I(x, y)
$$

The second stage is detecting keypoints. Keypoint determination takes a sample point that is compared with 26 pixels neighboring. If the point has the smallest (local minima) or largest (local maxima) value, the point will become a candidate keypoint. Candidate keypoints chosen are then filtered to eliminate low-contrast keypoints and keypoints located near the edge. Keypoints are also calculated on magnitude and angle. This stage makes SIFT invariant orientation.

In creating descriptors on the keypoint, the SIFT algorithm creates 16x16 pixel size around the keypoint and $4 \times 4$ sub-areas with eight orientation directions. The final result is 128 descriptors.

\section{AKAZE algorithm}

The AKAZE algorithm consists of 4 parts: computing the contrast factor, constructing nonlinear scale-space, detecting features, and creating descriptors [10]. The flowchart of the AKAZE algorithm is shown in Figure 4. The first stage is computing the contrast factor [14]. A Gaussian filter smoothes the query image or frame video. The next step is calculating the maximum absolute gradient value $\left(h_{\max }\right)$. Index $i$ is looping on the histogram. Afterward, the gradient value is divided by a histogram of 300 bins. The formula to compute the contrast factor $k$ is expressed in (2).

$$
k=\frac{h_{\max } \cdot i}{300}
$$

The second stage is constructing a nonlinear scalespace [15]. The scale-space approach is as same as the SIFT algorithm, which discretizes the scale-space in logarithmic steps arranged in octaves and scales. The scale-space in the AKAZE algorithm is a pyramid which is shown in Figure 5. It consists of sub-levels that each octave is quarter size than the previous octave [16].

The third step is the feature detector. The AKAZE algorithm uses the determinant of Hessian (DoH) blobdetector. After constructing the nonlinear scale-space, DoH query image or video frame is computing at sublevels increase. The keypoints or features in the query image or frame video are extracted by comparing the $\mathrm{DoH}$ image with the neighboring window of size $3 \times 3$.

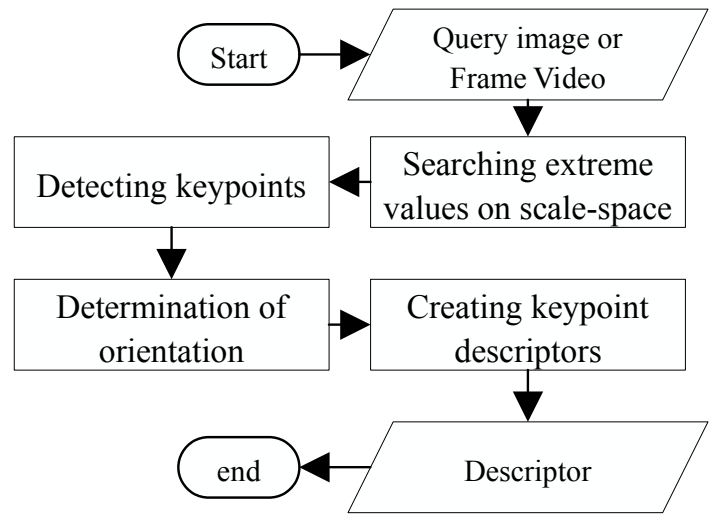

Figure 3. The flowchart of the SIFT algorithm

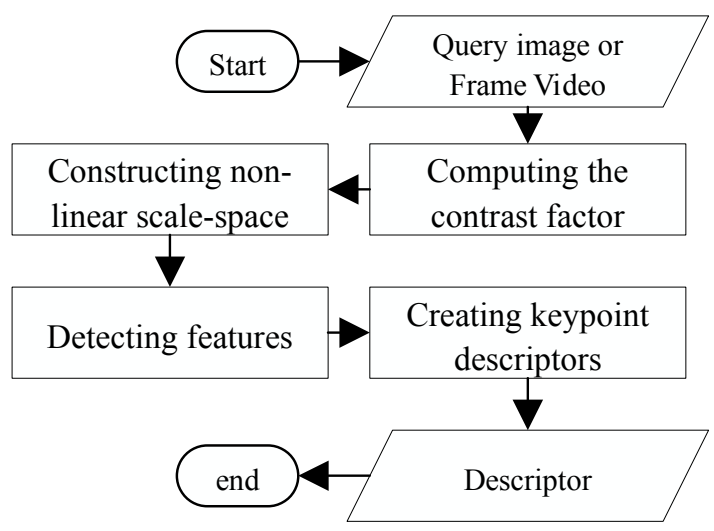

Figure 4. The flowchart of the AKAZE algorithm

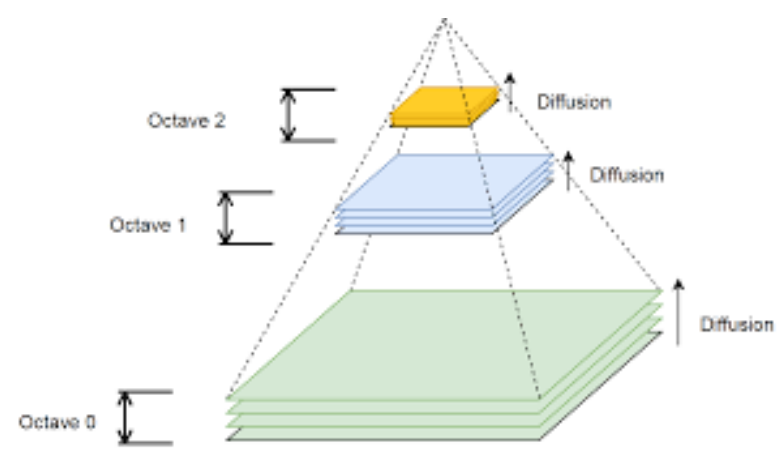

Figure 5. Scale-space representation [16]

The pixel point is compared with eight neighbors. If it is greater than eight neighbors, then it becomes a keypoint.

The next step is creating a descriptor [17]. AKAZE algorithm generates a descriptor on each keypoint that scales and rotates invariant. Each keypoint is made by sampling $16 \times 16$ pixels around the keypoint and dividing it into $4 \times 4$ blocks. The histogram is then calculated by eight bins. The final result is 128 descriptors of the AKAZE algorithm.

\section{Matching feature query image with frame video}

The SIFT algorithm uses the FLANN method in the matching feature stage, while the AKAZE algorithm uses 


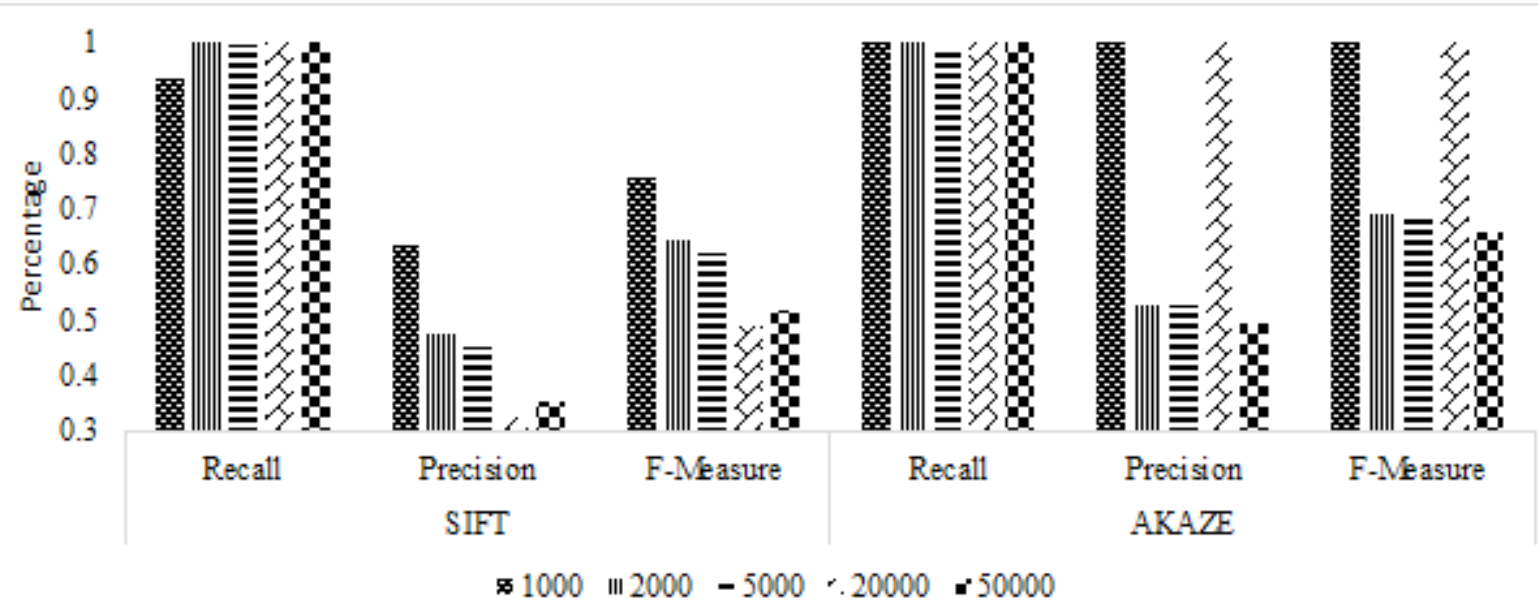

Figure 6. The performance of the SIFT and AKAZE on the full HD videos

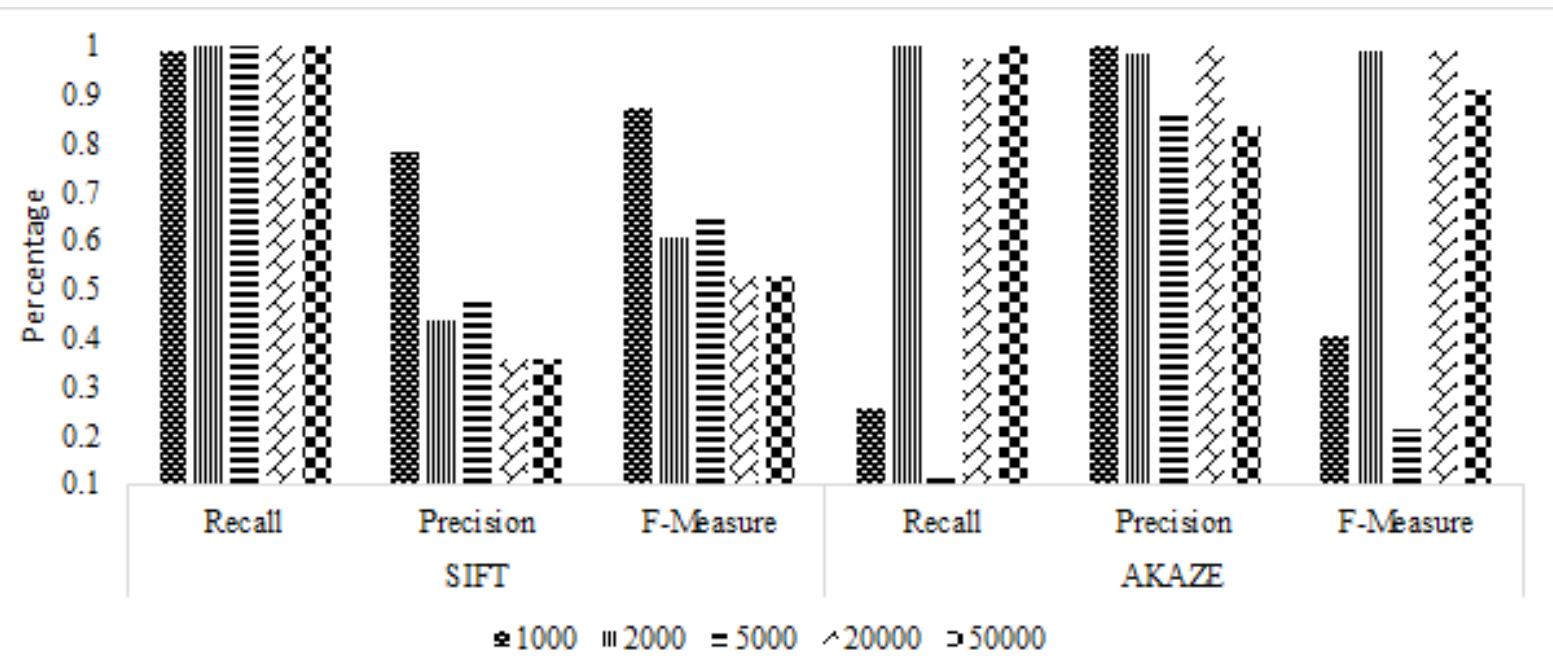

Figure 7. The performance of the SIFT and AKAZE on the HD videos

BF-Matcher. The requirement of matching features is at least four keypoints having good matches on query image with frame video. If good matches are more than or equal to four, a Homography matrix search of the query image and frame video is performed [18]. The object on an image will have geometrical transformations such as translation, rotation, scaling, and shear. The next stage is checking whether the Homography matrix is formed or not. The process will be terminated if the Homography matrix is not formed, which indicates a mismatch.

The Fast Library Approximated Nearest Neighbor (FLANN) method is used to find the nearest neighbor's value [19]. The SIFT algorithm produces 128 dimensions of descriptor for each keypoint. Therefore, matching features with $\mathrm{K}-\mathrm{NN}$ is considered inefficient, so the FLANN method for matching multi-dimensional data is needed. The FLANN method uses the K-Dimensional Tree (KD-Tree) to represent multi-dimensional binary tree data to separate certain areas based on their value position [20].

The AKAZE algorithm generates keypoints and binary descriptors in the query image and frame video. The BF-Matcher work compares each query image descriptor with all frame video descriptors to find the smallest result [21].

\section{RESULTS AND DISCUSSION}

\section{A. The experiment of recording resolution}

Video resolution needs to be tested to see which resolution produces the best $F 1$ value. Resolution experiments use Full HD (1920x1080) and HD (1280x720) resolutions. In this experiment, the distance between the currency object and the camera is $20 \mathrm{~cm}$. Figure 6 and Figure 7 present the resulting graph of the $F 1$ value on the Full HD and HD videos, respectively.

In Figure 6, the AKAZE algorithm gives better results than the SIFT algorithm on all currency experiments. Moreover, on currencies 1000 and 20000, the AKAZE algorithm gets the maximum value of $F 1$. In currencies 2000, 5000, and 50000, many false positive were found, which were negative data but were recognized as positive by the system. For example, testing the 50000 currency on the video often matches the query image of 5000. This false positive recognition 


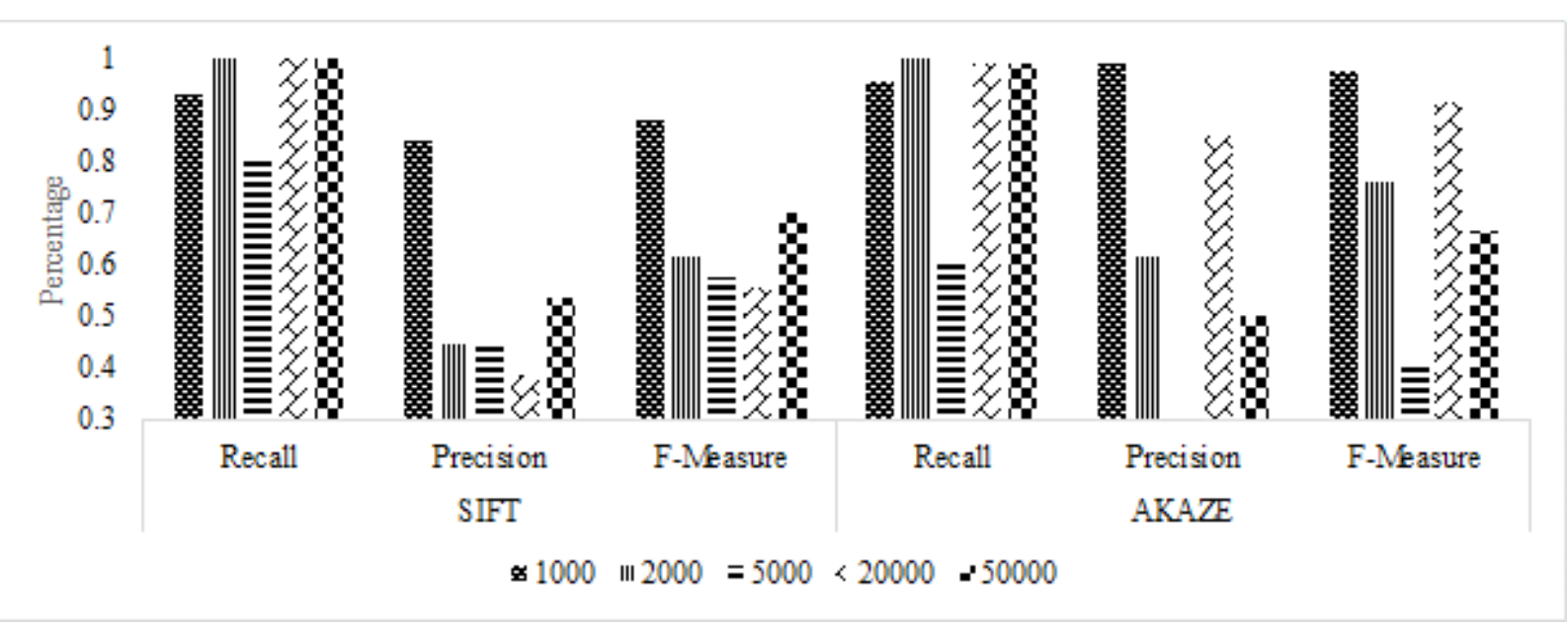

Figure 8. The performance of the SIFT and AKAZE at a distance of $10 \mathrm{~cm}$

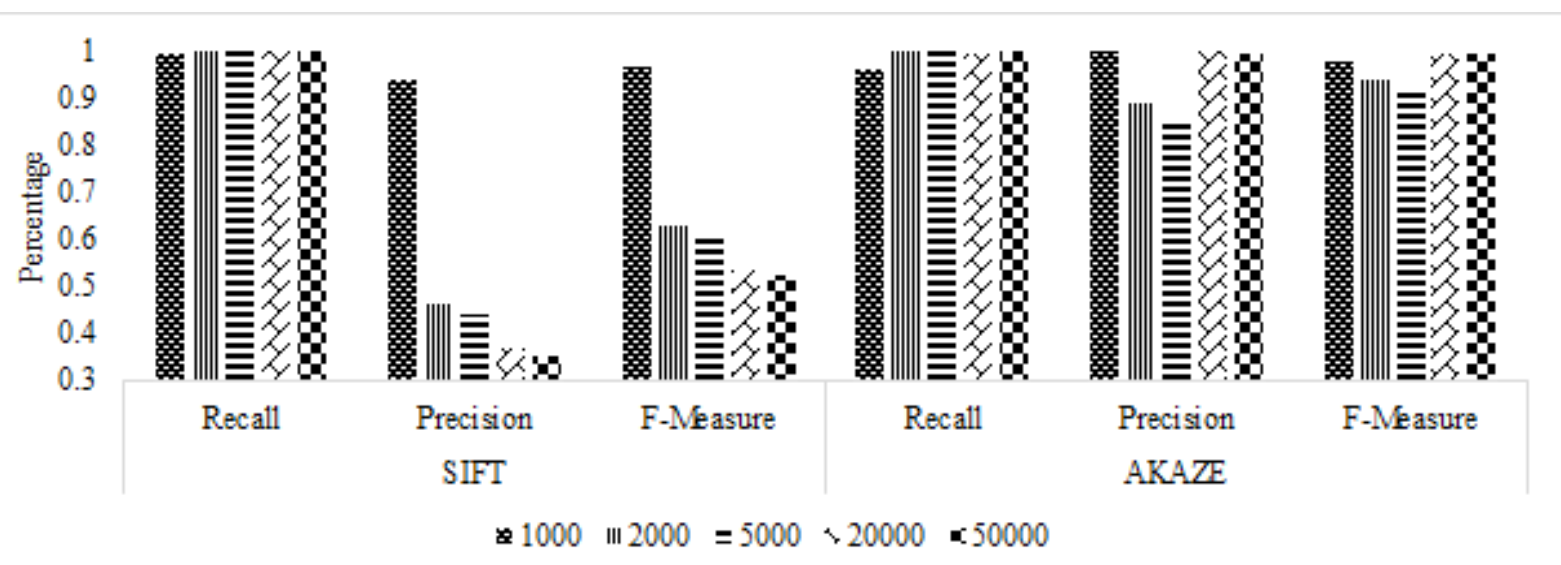

Figure 9. The performance of the SIFT and AKAZE at a distance of $30 \mathrm{~cm}$

is because there is the same leading number, which is 5 . In Naharul et al. [22], there was also an error in detecting the same nominal using the template matching technique. It is because the numbers 5000 and 50000 only add 0 on the last digit of the nominal.

The recall-precision curves in Figure 6 show that the SIFT algorithm has a lower precision value than the AKAZE algorithm. This low precision value indicates that the level of accuracy between the information requested by the user and the answer by the system is often wrong. Both Full HD and HD resolutions produce good recall values. In various experiments, the recall and precision values are inversely related in various experiments. If the recall value is high, the precision is likely low [23].

In the HD resolution experiment, as shown in Figure 7, the average matching result of the AKAZE algorithm is better than the SIFT algorithm. The $F 1$ value on the 1000 and 5000 currencies is low because the resolution of the recording video is very influential. In Adhinata et al. [24], video resolution also significantly affects the object detection results and speed. Decreasing the resolution results in fewer features being detected so that false negatives, which means positive data are recognized as negative by the system, often occur. For example, video data that use the currency of 5000 and is matched against a query image of 5000 , the results do not match.
However, compared to the average resolution of Full HD and HD, Full HD resolution produces an $F 1$ average value of 0.81 , which is better than $\mathrm{HD} 0.70$ for the AKAZE algorithm. The average SIFT algorithm tends to be the same in Full HD and HD resolutions, namely 0.60 and 0.63 . Therefore, in the currency distance experiment with the camera using Full HD resolution.

In Meharu and Worku [11], the use of deep learning was quite accurate, reaching $F 1$ of 0.918 . Meanwhile, this research uses five currencies, each with a currency of 1700 , so that the total training data is 8500 images. This training process takes a long time, which is 48 hours. On the other hand, our proposed research does not go through a training process and uses five different nominal currencies. The experimental results in this study are also completely accurate, where the $F 1$ value is 0.81 on the use of Full HD video data.

\section{B. The optimal distance for object matching}

The experiment on the distance of the currency object with a camera aims to determine the optimal matching distance. This experiment uses Full HD resolution, considering that Full HD resolution resulted in an optimal value of $F 1$. The distance variation in this experiment uses a distance of $10 \mathrm{~cm}$, and $30 \mathrm{~cm}$. Figure 8 and 

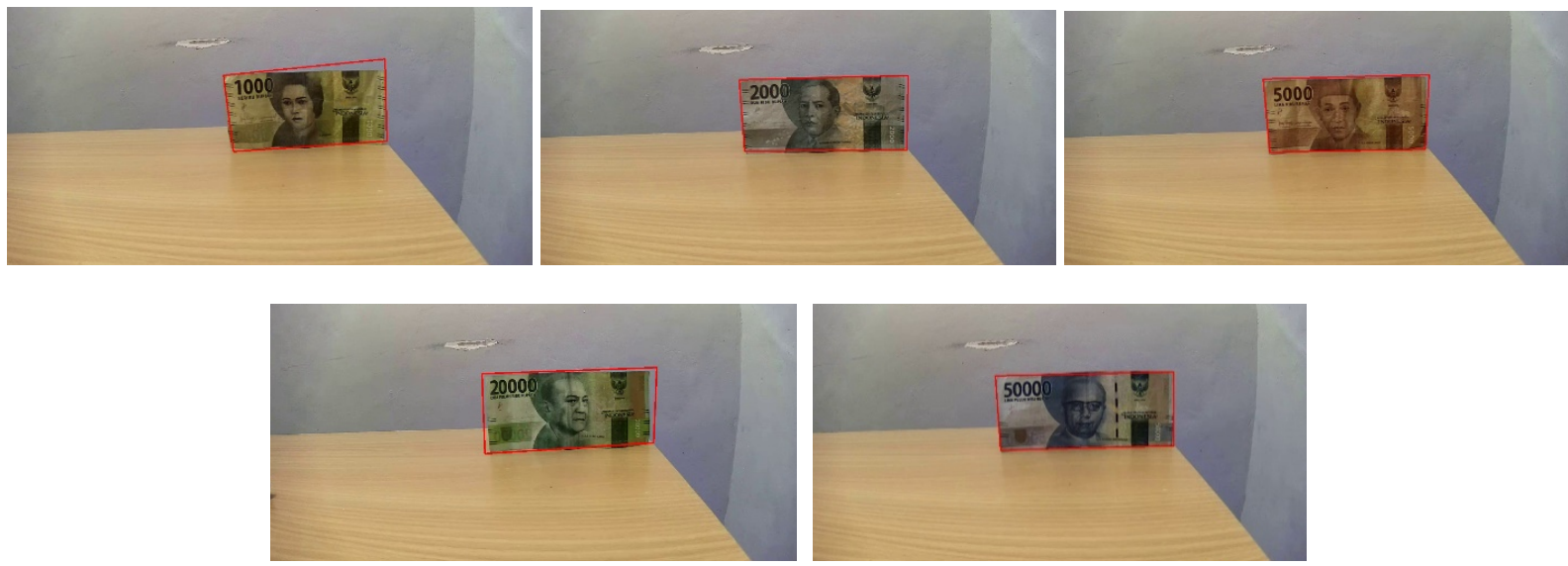

Figure 10. The result of matching query image and video

Figure 9 show the effect of a distance of $10 \mathrm{~cm}$ and 30 $\mathrm{cm}$ on the matching results.

The AKAZE algorithm optimally results in an average $F 1$ value of 0.97 at $30 \mathrm{~cm}$. However, in the SIFT algorithm, the experimental results showed no significant changes in the $10 \mathrm{~cm}$ or $30 \mathrm{~cm}$ distance with an average $F 1$ value of 0.66 and 0.63 . At $10 \mathrm{~cm}$, the $F 1$ value is not optimal because the object is too close to the camera, which causes the feature size to be too large compared to the features in the query image.

The use of this template matching technique dramatically affects the distance. Features that are too large cause the currency object to go undetected. It is because the template matching technique uses feature similarity in the query image. Adhinata et al. [25] analyzed the object's distance with the camera, which significantly affected accuracy results. Video data that is too large or small makes it incompatible with the query image because its features have a low level of similarity. Therefore, in studies that use distance variations, the optimal results obtained are at a distance of $30 \mathrm{~cm}$ with an $F 1$ value of 0.97 .

\section{Discussion}

Based on the experiment of resolution and distance, the AKAZE algorithm produces a better $F 1$ value than the SIFT algorithm. In terms of processing speed on each frame, the AKAZE algorithm was found to be faster than the SIFT algorithm, as shown in Table 2. This enables the real-time processing of video data. In a $\mathrm{Xu}$ et al. [4], the speed of the SIFT algorithm was 0.59 seconds. However, this speed is also influenced by the computer hardware used.

The AKAZE algorithm takes 0.25 seconds to process a single frame at Full HD resolution. It is faster than the SIFT algorithm. Processing real-time video data can be done by selecting keyframes, such as processing only a sequence of frames. The data processing speed is highly dependent on the resolution of the video data. The use of full HD data at a distance of $30 \mathrm{~cm}$ produces an optimal $F 1$ value in all currencies, which is more than 0.9 , with an average of 0.97 . However, the Full HD resolution makes processing only four fps in the real-time video data
Table 2. Time processing on matching per frame

\begin{tabular}{ccc}
\hline \multirow{2}{*}{ Resolution } & \multicolumn{2}{c}{ Speed of processing frame (second) } \\
\cline { 2 - 3 } & SIFT & AKAZE \\
\hline Full HD & 0.305 & 0.251 \\
HD & 0.154 & 0.113 \\
\hline
\end{tabular}

processing. In contrast, HD resolution video data can produce nine fps but an $F 1$ value of 0.7 . Overall, the processing speed of AKAZE video frames is faster than SIFT, both at Full HD and HD resolutions.

The weakness of this research is mainly on the value of F1 currency objects which have the same nominal value on the front number. Our future work will modify the recognition of nominal currency numbers to improve accuracy in HD resolution. The results of matching the query image and video data are shown in Figure 10.

\section{CONCLUSION}

The AKAZE algorithm for currency recognition gives the $F 1$ value of 0.97 and a speed of 0.251 at Full HD resolution better than the SIFT. The processing speed of AKAZE video frames is also faster than the SIFT, both at full HD and HD resolutions. Future research can modify the feature extraction method section to make it more accurate when using HD resolution. In this research, experiments at HD resolution resulted in a processing speed of 0.113 , but the matching results were not quite accurate.

\section{REFERENCES}

[1] G. Farooque, A. B. Sargano, I. Shafi, and W. Ali, "Coin recognition with reduced feature set sift algorithm using neural network," in the 14th International Conference on Frontiers of Information Technology, Islamabad, Pakistan, Dec. 2016, pp. 93-98. doi: 10.1109/FIT.2016.025

[2] B. Jiang, X. Li, L. Yin, W. Yue, and S. Wang, "Object recognition in remote sensing images using combined deep features," in the 3rd Information Technology, Networking, Electronic 
and Automation Control Conference, Chengdu, China, Mar. 2019, pp. 606-610. doi: 10.1109/ITNEC.2019.8729392

[3] Y. Zhang and J. Liang, "A vision based method for object recognition," in the 3rd International Conference on Information Science and Control Engineering, Beijing, China, Jul. 2016, pp. 139142. doi: 10.1109/ICISCE.2016.40

[4] J. Xu, G. Yang, Y. Liu, and J. Zhong, "Coin recognition method based on SIFT algorithm," in the 4th International Conference on Information Science and Control Engineering, Changsha, China, Jul. 2017, pp. 229-233. doi: 10.1109/ICISCE.2017.57

[5] A. Kuznetsov and A. Savchenko, "Sport teams logo detection based on deep local features," in International Multi-Conference on Engineering, Computer and Information Sciences, Novosibirsk, Russia, Oct. 2019, pp. 548-552. doi: 10.1109/SIBIRCON48586.2019.8958301

[6] N. Dalal and B. Triggs, "Histograms of oriented gradients for human detection," Lecture Notes in Computer Science, vol. 9284, pp. 498-515, 2015. doi: 10.1007/978-3-319-23528-8_31

[7] D. G. Lowe, "Distinctive image features from scale-invariant keypoints," International Journal of Computer Vision, vol. 60, no. 2, pp. 91-110, 2004. doi: 10.1023/B:VISI.0000029664.99615.94

[8] H. Bay, A. Ess, T. Tuytelaars, and L. Vangool, "Speeded-Up Robust Features (SURF)," Computer Vision and Image Understanding, vol. 110, no. 3, pp. 346-359, 2006. doi: 10.1016/j.cviu.2007.09.014

[9] E. Rublee, V. Rabaud, K. Konolige, and G. Bradski, "ORB: An efficient alternative to SIFT or SURF," in the IEEE International Conference on Computer Vision, Barcelona, Spain, Nov. 2011, pp. 2564-2571. doi: 10.1109/ICCV.2011.6126544

[10] P. F. Alcantarilla, J. Nuevo, and A. Bartoli, "Fast explicit diffusion for accelerated features in nonlinear scale spaces," in the British Machine Vision Conference, Bristol, UK, Sep. 2013, pp. 1-9. doi: $10.5244 /$ C.27.13

[11] M. L. Meharu and H. S. Worku, "Real-Time Ethiopian currency recognition for visually disabled peoples using convolutional neural network," Research Square, preprint, pp. 1-24, 2020. doi: 10.21203/rs.3.rs-125061/v1

[12] Q. Zhang, W. Q. Yan, and M. Kankanhalli, "Overview of currency recognition using deep learning," Journal of Banking and Financial Technology, vol. 3, no. 1, pp. 59-69, 2019. doi: 10.1007/s42786-018-00007-1

[13] D. Henry, Y. Yao, R. Fulton, and A. Kyme, "An optimized feature detector for markerless motion tracking in motion-compensated neuroimaging," in the IEEE Nuclear Science Symposium and Medical Imaging Conference, Atlanta, USA, Oct. 2017, pp. 1-4. doi: 10.1109/NSSMIC.2017.8532865
[14] P. Soleimani, D. W. Capson, and K. F. Li, "Realtime FPGA-based implementation of the AKAZE algorithm with nonlinear scale space generation using image partitioning," Journal of Real-Time Image Processing, vol. 18, pp. 2123-2134, 2021. doi: 10.1007/s11554-021-01089-9

[15] H. Seong, H. Choi, H. Son, and C. Kim, "Imagebased 3D building reconstruction using A-KAZE feature extraction algorithm," in the International Symposium on Automation and Robotics in Construction, Berlin, Germany, Jul. 2018. doi: 10.22260/isarc2018/0127

[16] L. Kalms, K. Mohamed, and D. Göhringer, "Accelerated embedded AKAZE feature detection algorithm on FPGA," in ACM International Conference Proceeding Series, Bochum, Germany, Jun. 2017, pp. 3-8. doi: 10.1145/3120895.3120898

[17] B. Soni, V. Anji Reddy, N. B. Muppalaneni, and C. Lalrempuii, "Image forgery detection using AKAZE keypoint feature extraction and trie matching," International Journal of Innovative Technology and Exploring Engineering, vol. 9, no. 1, pp. 2208-2213, 2019. doi: 10.35940/ijitee.A4784.119119

[18] D. K. Iakovidis, E. Spyrou, and D. Diamantis, "Efficient homography-based video visualization for wireless capsule endoscopy," in the IEEE International Conference on BioInformatics and BioEngineering, Chania, Greece , Nov. 2013, pp. 1-4. doi: 10.1109/BIBE.2013.6701598

[19] M. Muja and D. Lowe, "FLANN - Fast Library for Approximate Nearest Neighbors: User manual," Univ. of British Columbia, Canada, pp. 1-21, 2009.

[20] J. Jo, J. Seo, and J. D. Fekete, "PANENE: A Progressive Algorithm for Indexing and Querying Approximate k-Nearest Neighbors," IEEE Transactions on Visualization and Computer Graphics, vol. 26, no. 2, pp. 1347-1360, 2020. doi: 10.1109/TVCG.2018.2869149

[21] I. W. A. Suryawibawa, I. K. G. D. Putra, and N. K. A. Wirdiani, "Herbs recognition based on Android using OpenCV," International Journal of Image, Graphics and Signal Processing, vol. 7, no. 2, pp. 1-7, 2015. doi: 10.5815/ijigsp.2015.02.01

[22] M. Naharul, H. Najihul, and S. Adinugroho, "Implementasi metode template matching untuk mengenali nilai angka pada citra uang kertas yang dipindai," Jurnal Pengembangan Teknologi Informasi dan Ilmu Komputer, vol. 3, no. 2, pp. 1550-1556, 2019.

[23] N. P. Lestari, "Uji recall and precision sistem temu kembali informasi OPAC Perpustakaan ITS Surabaya," B.Eng thesis, Universitas Airlangga, Surabaya, Indonesia, 2016.

[24] F. D. Adhinata, M. Ikhsan, and W. Wahyono, "People counter on CCTV video using histogram of oriented gradient and Kalman filter methods," Jurnal Teknologi dan Sistem Komputer, vol. 8, no. 3, pp. 222-227, 2020. doi: 10.14710/jtsiskom.2020.13660 
[25] F. D. Adhinata, A. Harjoko, and Wahyono, "Object searching on video using orb descriptor and support vector machine," in Advances in
Computational Collective Intelligence, Da Nang, Vietnam, Nov. 2020, pp. 239-251. doi: 10.1007/978-3-030-63119-2 20

(c) (i) (2) (C2021. This open-access article is distributed under the terms and conditions of the Creative cc) Commons Attribution-ShareAlike 4.0 International License. 